\title{
Power system security analysis under transmission line outage condition
}

\author{
S. Ravindra ${ }^{1}$, V. C. Veera Reddy ${ }^{2}$, S. Sivanagaraju ${ }^{3}$ \\ Associate professor, Department of Electrical and Electronics Engineering, QISCET, Ongole, India ${ }^{1}$ \\ Principal and Professor, Department of Electrical and Electronics Engineering, AITS, Tirupati, India ${ }^{2}$ \\ Professor, Department of Electrical and Electronics Engineering, UCEK, JNTUK, Kakinada, India ${ }^{3}$
}

\begin{abstract}
The power system becomes more severe under contingency conditions. In general, the contingencies may be outage of transmission lines or generators. To identify the effect of outages on system security, the contingency analysis is one of the analytical tools. The congestion on a system must be manages using generation rescheduling and with the proper control of the compensation equipments. The transmission line outage sometime increases the total power system losses, cascading outage of transmission lines, etc. Finally, the system leads to collapse condition. To avoid this type of uncontrolled condition, the power system security needs to be analyzed under transmission line outage condition. In this paper, the most critical transmission lines are identified using line collapse proximity index values and the system severity is analyzed in terms of transmission line loadings, bus voltage magnitude variations are tested on standard IEEE-14 bus test system and the analytical results are presented in numerically and as well as graphically.
\end{abstract}

Keywords: Power system security, contingency analysis, transmission line outages, line collapse proximity index.

\section{INTRODUCTION}

Now a day, because of the restructuring and deregulation and generating units. In [6], the potential benefits provided of the power system network, the operation, control and by demand-side management programs applied in power management of the system becomes one of the most system congested areas to improve voltage security are challenging tasks. To avoid uncontrolled operation of investigated in this congested areas are indicated by power system, it is necessary to analyse the severity of expanded modal analysis technique from a new system under contingency conditions. There are different perspective of active power variations.

types of contingency conditions that lead the system to From the careful review of the literature, it is identified collapse condition.

In contingency analysis, outage of transmission lines and ranking processes are performed to determine the most severe line outage contingencies considering line overloads and bus voltage limit violations through performance index. An important issue of transmission system congestion management in a pool electricity market environment with the consideration of voltage stability as loadability limit is present in [1]. In this, the optimal generators rescheduling has been obtained for three block bid structure submitted to the ISO in a day-ahead market.

that transmission line outage condition is the most severe contingency condition in power system. Hence, it is necessary to analyse the severity of the system under this contingency condition. In this paper, the most critical transmission lines in a given system are identified using line collapse proximity index (LCPI) values.The complete methodology of identifying system severity in terms of transmission line loadings and bus voltage magnitude violations are tested on standard IEEE-14 bus system with supporting numerical and as well as graphical results.

\section{CONTINGENCY ANALYSIS}

In [2], an attempt on congestion management by The system severity should be evaluated for one of the considering the impact of constant current, impedance, and severe conditions such as contingency condition. The power (ZIP) load model along with the load variation result of this analysis allows the system to be operated pattern in a day-a-head hybrid electricity market. In [3], securely. Now a day, because of the modernization of the methods to the relieve congestion by generation power system with the help of computers, it is possible to rescheduling and/or load shedding under sever congestion identify the most critical transmission lines [7] that causes are presented. A contingency analysis is performed in [4] the system to be operates in an uncontrolled manner and to detect and rank the faulted contingencies on the basis of leads cascading outages and failures. From this, it is their severity in electrical energy transmission systems. necessary to perform a contingency analysis to Minimization of the average loadability on all energy predetermine the system effectiveness before they arise. transmission lines minimizes the active power losses under As the present power system is an interconnected network, pre-contingency conditions.

In [5], they proposed a stochastic programming framework for congestion management considering the power system uncertainties comprising outage of transmission branches

when transmission lines are lost, due to which some of the transmission lines get overloaded [8]. From this discussion, it is necessary an operational person should know the information regarding the severity of the 
transmission lines and the possibilities of the generator outages to maintain the system secure. In common practice, contingency analysis is performed on a given system with single outage events (one transmission line outage or one generator outage) or multiple outage events (two/more transmission lines outage or two/more generators outage or combinations thereof) are analyzed. For each of the events, the limit violations of power flows in transmission lines and voltage magnitude deviations at buses are identified. Finally, the event which has highest severity is identified and the respective precautions are suggested to improve the system security for safer operation.

\section{III.POWER SYSTEM SECURITY}

In general, the main aim of power system operation and control is to meet the demand continuously without any failures. While, in this operation, sometimes, outage of generator due to failure of the auxiliary equipment or removal of a transmission line for maintenance purpose or due to storm and other effects may happens. Due to which, the system frequency may drops and leads to load shedding or uncontrolled operation and sometimes leads to system collapse condition [9]. This happens mainly due to the overloading of the transmission lines, voltage deviation at the load buses and lack of reactive power support at the load buses.

\section{A. Line collapse proximity index}

From the literature, it is identified that, there are variety of indices to identify the most severe lines in a given system. Due to the difficulties reported in the literature for the available indices, a new index based on the effect of power flow in transmission lines, line charging reactance and the direction of reactive power flow with respect of the direction of active power flow known as "Line collapse proximity index" (LCPI) is proposed [10]. The modeling of this index is based on the exact pi-model of the transmission lines using ABCD-parameters.

Conventionally, the relation between system parameters for a transmission line connected between buses ' $s$ ' and ' $r$ ' can be expressed as follows

$$
\left[\begin{array}{l}
\bar{V}_{s} \\
\bar{I}_{s}
\end{array}\right]=\left[\begin{array}{ll}
A & B \\
C & D
\end{array}\right]\left[\begin{array}{l}
\bar{V}_{r} \\
\bar{I}_{r}
\end{array}\right]
$$

Here, A, B, C and D are the transmission line parameters related to two-port network and can be expressed as $A=\left(1+\frac{Z Y}{2}\right)$-Open circuit reverse voltage transfer ratio $B=Z$-Short circuit reverse transfer impedance $C=Y\left(1+\frac{Z Y}{4}\right)$-Open circuit reverse transfer admittance $D=A$-Short circuit reverse current transfer ratio Here, $\mathrm{Z}$ and $\mathrm{Y}$ are the transmission line impedance and line charging admittance respectively. $\bar{V}_{s}, \bar{V}_{r}$ and $\bar{I}_{s}, \bar{I}_{r}$ are the voltage and current vectors at the respective buses. The current at the receiving end of the transmission line can be calculated as

$$
\begin{aligned}
\bar{S}_{r} & =\bar{V}_{r}\left(\bar{I}_{r}\right)^{*} \\
\bar{I}_{r}=\frac{\left(\bar{S}_{r}\right)^{*}}{\left(\bar{V}_{r}\right)^{*}} & =\frac{P_{r}-j Q_{r}}{\left(\bar{V}_{r}\right)^{*}}=\frac{P_{r}-j Q_{r}}{V_{r} \angle-\delta_{r}}
\end{aligned}
$$

(3) Step 5: Calculate line collapse proximity index (LCPI) values for each of the transmission lines.

Step 6: Rank the lines based on their LCPI values.

Where, $P_{r}, Q_{r}$ are the active and reactive powers and $V_{r}, \delta_{r}$ are the voltage magnitude and voltage angles at the receiving end respectively.

From Eqn (1), the sending end voltage of the transmission line can be expressed as

$$
V_{s} \angle \delta_{s}=A \angle \alpha V_{r} \angle \delta_{r}+B \angle \beta I_{r} \angle 0^{0}
$$

Here, $\mathrm{A}$ and $\mathrm{B}$ are magnitudes and $\alpha, \beta$ are the phase angles of parameters of A, B respectively.

After solving Eqn (4) using Eqn (3), we get

$$
V_{s} \angle \delta_{s}=A \angle \alpha V_{r} \angle \delta_{r}+B \angle \beta\left(\frac{P_{r}-j Q_{r}}{V_{r} \angle-\delta_{r}}\right)
$$

Rearranging Eqn (5), results

$$
V_{s} V_{r} \angle\left(\delta_{s}-\delta_{r}\right)=A \angle \alpha V_{r}^{2}+B \angle \beta\left(P_{r}-j Q_{r}\right)
$$

Let us define $\delta=\delta_{s}-\delta_{r}$ and solve for Eqn (6), we get $V_{s} V_{r} \cos \delta+j V_{s} V_{r} \sin \delta=A V_{r}^{2} \cos \alpha+j A V_{r}^{2} \sin \alpha+$ $B\left(P_{r} \cos \beta+Q_{r} \sin \beta\right)-j B\left(Q_{r} \cos \beta-P_{r} \sin \beta\right)$

The real part of Eqn (7) can be written as

$$
V_{s} V_{r} \cos \delta=A V_{r}^{2} \cos \delta+B\left(P_{r} \cos \beta+Q_{r} \sin \beta\right)
$$

Eqn (8) seems to be the quadratic equation and the roots of this equation results the receiving end voltage, this can be derived as

$V_{r}=\frac{-V_{s} \cos \delta \pm \sqrt{\left(V_{s} \cos \delta\right)^{2}-4 A \cos \alpha\left(P_{r} B \cos \beta+Q_{r} B \sin \beta\right)}}{2 A \cos \alpha}(9)$ The real and non-zeros values of the Eqn (9) can be obtained by equating determinant of Eqn (9) to greater than zero, i.e.

$\left(V_{s} \cos \delta\right)^{2}-4 A \cos \alpha\left(P_{r} B \cos \beta+Q_{r} B \sin \beta\right)>0(10)$ Based on this, the condition that should satisfy to operate power system securely to avoid voltage collapse condition can be expressed as

$$
\frac{4 A \cos \alpha\left(P_{r} B \cos \beta+Q_{r} B \sin \beta\right)}{\left(V_{s} \cos \delta\right)^{2}}<1
$$

From this, the line collapse proximity index for each of the transmission lines can be calculated using

$$
L C P I=\frac{4 A \cos \alpha\left(P_{r} B \cos \beta+Q_{r} B \sin \beta\right)}{\left(V_{s} \cos \delta\right)^{2}}
$$

For secured operation, the value of LCPI must be less than 1 (one).

\section{IV.ALGORITHM FOR SECURITY ANALYSIS UNDER TRANSMISSION LINE OUTAGE CONDITION}

It is necessary to predetermine the most severe transmission lines in a system to analyze the system security. To suggest and to take the protective corrections against contingency conditions, it is necessary to analyze the system severity under contingency conditions. For this purpose, in this paper, $15 \%$ of the transmission line outages are considered to form contingency condition.

To identify the severity of transmission line outages on system security, the steps needs to be followed are:

Step 1: Read the system line and bus data.

Step 2: Perform load flow analysis using Newton-Raphson method.

Step 3: Obtain voltage magnitudes at all load buses and voltage angles at all buses except slack bus.

Step 4: Calculate power flows in each of the transmission lines.

JIREEICE.2015.3109 
Step 7: Remove the top $15 \%$ severe lines from a given system to create contingency condition.

Step 8: Repeat steps from 2 to 4 and identify the over loaded lines and voltage violated buses to analyze the system security.

\section{RESULTS AND ANALYSIS}

IEEE-14 bus system with twenty transmission lines, five generators, three tap-changing transformers and one shunt compensator is considered. The proposed methodology is tested for the following condition.

$>\quad$ Identifying critical lines using LCPI values and outage of top $15 \%$ LCPI valued lines to create transmission line contingency condition.

For this system, by following the procedure given in section-3, LCPI value for each of the transmission lines is calculated. Here, LCPI values are not calculated for five lines i.e. line- 1 connected between buses 1 and 2 is double circuit line, lines 8, 9, 10 are transformer connected lines and line-14 isolates the generator from system. Hence, for this system LCPI values for fifteen transmission lines out of twenty are calculated and tabulated in Table.1. Based on these LCPI values, respective ranks are assigned to each of the lines. From Table.1, it is observed that, line-6 connected between buses 3 and 4 is assigned as rank-1 and line- 17 connected between buses 9 and 14 is assigned as rank-2. To analyze the system security under transmission line outage condition, the procedure given in section 4 is followed. For this, the top $15 \%$ of the critical lines i.e. lines which are ranked 1 and 2 are removed from the system to create contingency condition. The Variation of LCPI values is shown in Fig. 1.

TABLE. 1 LCPI VALUES FOR IEEE-14 BUS SYSTEM

\begin{tabular}{|c|c|c|c|c|}
\hline $\begin{array}{c}\text { S. } \\
\text { No }\end{array}$ & Line No & $\begin{array}{c}\text { From bus } \\
\text {-To bus }\end{array}$ & $\begin{array}{c}\text { LCPI } \\
\text { value }\end{array}$ & Rank \\
\hline 1 & 2 & $1-5$ & 0.8147 & 7 \\
\hline 2 & 3 & $2-3$ & 0.9058 & 6 \\
\hline 3 & 4 & $2-4$ & 0.1270 & 14 \\
\hline 4 & 5 & $2-5$ & 0.9134 & 5 \\
\hline 5 & 6 & $3-4$ & 0.9706 & 1 \\
\hline 6 & 7 & $4-5$ & 0.0975 & 15 \\
\hline 7 & 11 & $6-11$ & 0.2785 & 12 \\
\hline 8 & 12 & $6-12$ & 0.5469 & 10 \\
\hline 9 & 13 & $6-13$ & 0.9275 & 3 \\
\hline 10 & 15 & $7-9$ & 0.6324 & 9 \\
\hline 11 & 16 & $9-10$ & 0.1576 & 13 \\
\hline 12 & 17 & $9-14$ & 0.9649 & 2 \\
\hline 13 & 18 & $10-11$ & 0.9172 & 4 \\
\hline 14 & 19 & $12-13$ & 0.4854 & 11 \\
\hline 15 & 20 & $13-14$ & 0.8003 & 8 \\
\hline
\end{tabular}

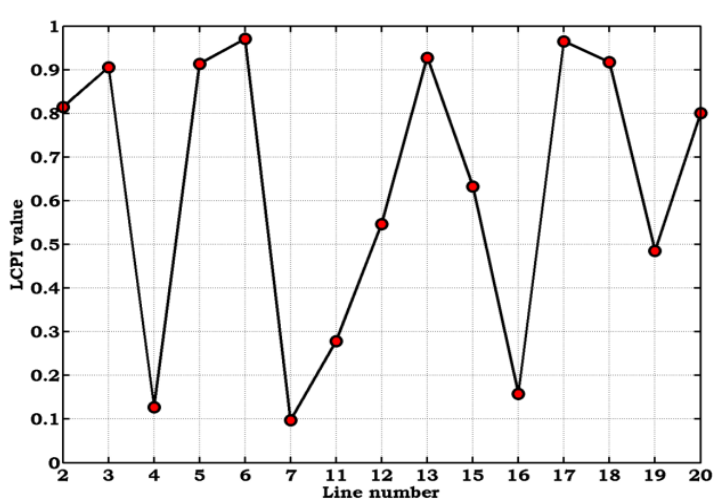

Fig.1. Variation of LCPI values

To show the effect of transmission line contingencies, the apparent power flow in lines under single and multi-line contingencies are tabulated in Table.2. From this table, it is identified that, line-15 is overloaded under the outage of first critical line i.e. line-6, this is because of the connection of line-15 is nearer to line- 6 . It is also identified that, lines 13 and 20 are overloaded under the outage of second critical line i.e. line-17, this is because of the connection of these lines is nearer to line-17. Finally, it is observed that, lines 13 and 20 are overloaded under the outage of lines 6 and 17; this is because of the nearer connection to the critical lines. The respective variation of power flow in lines is shown in Fig. 2.

TABLE.2. POWER FLOWS UNDER TRANSMISSION LINE(S) OUTAGE CONDITION

\begin{tabular}{|c|c|c|c|c|c|}
\hline \multirow{2}{*}{$\begin{array}{c}\text { Line } \\
\text { No }\end{array}$} & \multirow{4}{*}{$\begin{array}{c}\text { Normal } \\
\text { condition }\end{array}$} & \multicolumn{2}{|c|}{ Outage condition } & \multirow{2}{*}{ MVA } \\
\cline { 3 - 5 } & & Line-6 & $\begin{array}{c}\text { Line- } \\
\mathbf{1 7}\end{array}$ & $\begin{array}{c}\text { Lines } \\
\text { 6\&17 }\end{array}$ & \\
\hline 1 & 86.27 & 87.18 & 86.28 & 87.22 & 150 \\
\hline 2 & 48.43 & 47.40 & 48.87 & 47.80 & 85 \\
\hline 3 & 41.25 & 45.67 & 41.06 & 45.67 & 85 \\
\hline 4 & 41.42 & 39.34 & 41.12 & 38.99 & 85 \\
\hline 5 & 31.37 & 29.76 & 31.89 & 30.22 & 85 \\
\hline 6 & 8.80 & - & 9.52 & - & 85 \\
\hline 7 & 43.39 & 41.57 & 40.21 & 38.50 & 150 \\
\hline 8 & 20.10 & 19.89 & 18.44 & 18.08 & 30 \\
\hline 9 & 12.53 & 12.63 & 11.11 & 11.16 & 32 \\
\hline 10 & 30.69 & 30.94 & 34.90 & 35.19 & 45 \\
\hline 11 & 9.81 & 9.62 & 5.93 & 5.62 & 14 \\
\hline 12 & 8.38 & 8.35 & 10.44 & 10.44 & 32 \\
\hline 13 & 20.01 & 19.90 & 27.94 & 27.94 & 22 \\
\hline 14 & 19.29 & 18.60 & 17.40 & 16.59 & 32 \\
\hline 15 & 29.45 & 29.58 & 26.24 & 26.34 & 29 \\
\hline 16 & 5.88 & 6.15 & 8.81 & 9.12 & 32 \\
\hline 17 & 9.14 & 9.28 & - & - & 18 \\
\hline 18 & 5.82 & 5.68 & 1.96 & 1.64 & 12 \\
\hline 19 & 1.96 & 1.93 & 3.96 & 3.96 & 12 \\
\hline 20 & 7.01 & 6.90 & 16.41 & 16.41 & 12 \\
\hline
\end{tabular}




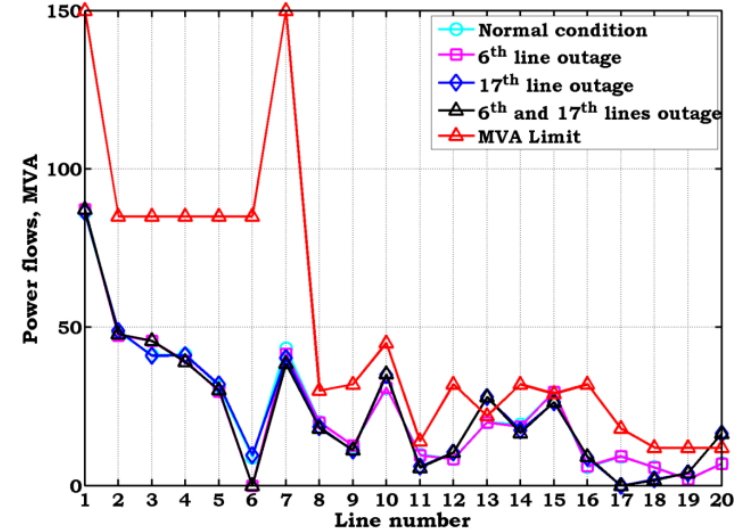

Fig.2. Variation of power flows under transmission line(s) outage condition

Voltage magnitude at buses under single and multi line contingencies are tabulated in Table.3. From this table, it is identified that, outage of multiple lines has more effect than that of single transmission line outage. The variation of voltage magnitude at buses under contingency conditions is shown in Fig.3.

TABLE.3. VOLTAGE MAGNITUDE AT BUSES UNDER TRANSMISSION LINE(S) OUTAGE CONDITION

\begin{tabular}{|c|c|c|c|c|}
\hline \multirow{2}{*}{$\begin{array}{c}\text { Bus. } \\
\text { No }\end{array}$} & $\begin{array}{c}\text { Vormal } \\
\text { Condition }\end{array}$ & $\begin{array}{c}\text { Outage } \\
\text { of } \\
\text { Line-6 }\end{array}$ & $\begin{array}{c}\text { Outage } \\
\text { of } \\
\text { Line-17 }\end{array}$ & $\begin{array}{c}\text { Outage of } \\
\text { Lines 6 \& } \\
\mathbf{1 7}\end{array}$ \\
\hline 1 & 1.060 & 1.060 & 1.060 & 1.060 \\
\hline 2 & 1.045 & 1.045 & 1.045 & 1.045 \\
\hline 3 & 1.010 & 1.010 & 1.010 & 1.010 \\
\hline 4 & 1.023 & 1.026 & 1.024 & 1.028 \\
\hline 5 & 1.025 & 1.027 & 1.026 & 1.028 \\
\hline 6 & 1.070 & 1.070 & 1.070 & 1.070 \\
\hline 7 & 1.063 & 1.064 & 1.066 & 1.068 \\
\hline 8 & 1.090 & 1.090 & 1.090 & 1.090 \\
\hline 9 & 1.055 & 1.056 & 1.062 & 1.064 \\
\hline 10 & 1.050 & 1.051 & 1.056 & 1.058 \\
\hline 11 & 1.056 & 1.057 & 1.060 & 1.061 \\
\hline 12 & 1.055 & 1.055 & 1.051 & 1.051 \\
\hline 13 & 1.050 & 1.050 & 1.041 & 1.041 \\
\hline 14 & 1.035 & 1.036 & 0.997 & 0.997 \\
\hline
\end{tabular}

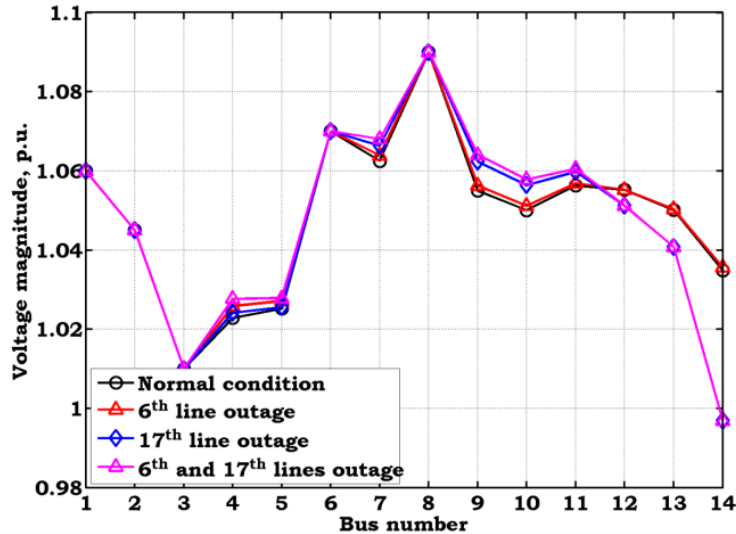

Fig.3. Variation of voltage magnitude at buses under transmission line(s) outage condition

\section{VI.CONCLUSION}

In this paper, the power system security has been analyzed under transmission line(s) outage condition. The most critical lines in a given system have been identified using line collapse proximity index values, and these lines are removed from the system to create contingency condition. The severity of this congestion has been analyzed in terms of transmission line loadings and bus voltage magnitude violations. From the analysis, it has been identified that, outage of two transmission lines has more effect on power flows when compared to outage of one of the transmission lines. The proposed methodology has been tested on standard IEEE-14 bus systems with supporting numerical and as well as graphical results.

\section{REFERENCES}

[1] Ashwani Kumar, Charan Sekhar,"Congestion management with FACTS devices in deregulated electricity markets ensuring loadability limit", Electrical Power and Energy Systems: 2013: 46:PP. 258-273.

[2] Ashwani Kumar, Ram Kumar Mittapalli, "Congestion management with generic load model in hybrid electricity markets with FACTS devices", Electrical Power and Energy Systems: 2014: 57: PP.49-63.

[3] Vijayakumar Krishnasamy, Jegatheesan, "Optimal location of multiple TCSCs for congestion management", Power Engineering and Optimization Conference (PEOCO), IEEE 7th International: 2013: PP.103-108.

[4] A. Lashkar Ara, J. Aghaei, M. Alaleh, H. Barati, "Contingencybased optimal placement of Optimal Unified Power Flow Controller (OUPFC) in electrical energy transmission systems", Scientia Iranica: 2013: 20: PP- 778-785.

[5] Masoud Esmaili, Nima Amjady, Heidar Ali Shayanfar, "Stochastic congestion management in power markets using efficient scenario approaches", Energy Conversion and Management: 2010:51: PP.2285-2293.

[6] Carolina M. Affonso, Luiz C.P. da Silva, "Potential benefits of implementing load management to improve power system security", Electrical Power and Energy Systems: 2010: 32: PP. 704-710.

[7] R.R. Matarucco, A. Bonini Neto, D.A. Alves, "Assessment of branch outage contingencies using the continuation Method", Electrical Power and Energy Systems: 2014: 55:PP.74-81.

[8] Salah Eldeen Gasim Mohamed, Abdelaziz Yousif Mohamed, Yousif Hassan Abdelrahim, "Power System Contingency Analysis to detect Network Weaknesses", Zaytoonah University International Engineering Conference on Design and Innovation in Infrastructure: 2012.

[9] Yesuratnam.G, Pushpa.M, "Congestion management for security oriented power system operation using generation rescheduling", Probabilistic Methods Applied to Power Systems (PMAPS): IEEE 11th International Conference on 2010: PP.287-292.

[10] Rajive Tiwari, K.R. Niazi, Vikas Gupta,"Line collapse proximity index for prediction of voltage collapse in power systems", Electrical Power and Energy Systems: 2012: 41:PP.105-111 .

\section{BIOGRAPHIES}

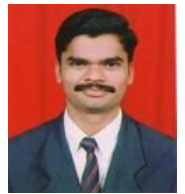

Sangu Ravindra received his B.Tech in Electrical Engineering from JNT University, Hyderabad \& M.E in Power Electronics \& Indstrial Drives from Satyabhama University, Chennai. $\mathrm{He}$ is currently pursuing Ph.D at JNT University, Kakinada. His research interest area is FACTS controllers, power system security, and Power quality.

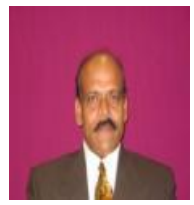

Dr.V.C.Veera Reddy received his B.Tech in Electrical Engineering from JNT University, Anantapur in 1979 \& M.Tech in Power System Operation \& Control 
from S.V University Tirupati, in 1981. He got Ph.D degree in Modeling \& Control of Load Frequency using new optimal control strategy from S.V. University Tirutati, in 1999.

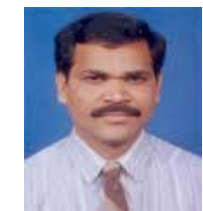

Dr. S.Sivanaga raju is Professor in the department of Electrical and Electronics Engineering, University College of Engineering Kakinada, Jawaharlal Nehru Technological University Kakinada, Kakinada, A.P., India. He completed his Master's degree from Indian Institute of Technology, Khargpur, India, in electrical power systems. He completed his doctoral program from Jawaharlal Nehru Technological University Hyderabad, Andhra Pradesh, India. His interests include FACTS Controllers, Electrical Distribution System Automation, Optimization Techniques, Voltage Stability, Power System Analysis, and Power System Operation and Control. 